\title{
Lachmann, Rainer
}

\section{Von der Fremdreligionen-Didaktik zum Interreligiösen Lernen}

Lachmann, Rainer [Hrsg.]; Rothgangel, Martin [Hrsg.]; Schröder, Bernd [Hrsg.]: Christentum und Religionen elementar. Lebensweltlich - theologisch - didaktisch. Göttingen : Vandenhoeck \& Ruprecht 2010, S. 26-40. - (Theologie für Lehrerinnen und Lehrer (TLL); 5)

Lachmann, Rainer: Von der Fremdreligionen-Didaktik zum Interreligiösen Lernen - In: Lachmann, Rainer [Hrsg.]; Rothgangel, Martin [Hrsg.]; Schröder, Bernd [Hrsg.]: Christentum und Religionen elementar. Lebensweltlich - theologisch - didaktisch. Göttingen : Vandenhoeck \& Ruprecht 2010, S. 26-40 - URN: urn:nbn:de:0111-pedocs-108585 - DOI: 10.25656/01:10858

http://www.v-r.de

\section{Nutzungsbedingungen}

Gewährt wird ein nicht exklusives, nicht übertragbares, persönliches und beschränktes Recht auf Nutzung dieses Dokuments. Dieses Dokument ist ausschließlich für den persönlichen, nicht-kommerziellen Gebrauch bestimmt. Die Nutzung stellt keine Übertragung des Eigentumsrechts an diesem Dokument dar und gilt vorbehaltlich der folgenden Einschränkungen: Auf sämtlichen Kopien dieses Dokuments müssen alle Urheberrechtshinweise und sonstigen Hinweise auf gesetzlichen Schutz beibehalten werden. Sie dürfen dieses Dokument nicht in irgendeiner Weise abändern, noch dürfen Sie dieses Dokument für öffentliche oder kommerzielle Zwecke vervielfältigen, öffentlich ausstellen, aufführen, vertreiben oder anderweitig nutzen.

Mit der Verwendung dieses Dokuments erkennen Sie die Nutzungsbedingungen an.

\section{Terms of use}

We grant a non-exclusive, non-transferable, individual and limited right to using this document

This document is solely intended for your personal, non-commercial use. Use of this document does not include any transfer of property rights and it is conditional to the following limitations: All of the copies of this documents must retain all copyright information and other information regarding legal protection. You are not allowed to alter this document in any way, to copy it for public or commercial purposes, to exhibit the document in public, to perform, distribute or otherwise use the document in public.

By using this particular document, you accept the above-stated conditions of use.

\section{Kontakt / Contact:}

\section{peDOcs}

DIPF | Leibniz-Institut für Bildungsforschung und Bildungsinformation Informationszentrum (IZ) Bildung

E-Mail: pedocs@dipf.de

Internet: www.pedocs.de

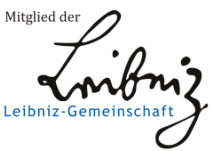




\author{
Rainer LAGHManN \\ Martin Rothgangel \\ BERND SGHRÖDER \\ (HG.)
}

\title{
CHRISTENTUM UND RELIGIONEN ELEMENTAR
}

Lebensweltlich - theologisch - didaktisch 


\title{
Theologie für Lehrerinnen und Lehrer (TLL)
}

\author{
Herausgegeben von \\ Rainer Lachmann und Gottfried Adam
}

\section{Band 5}

Mit 4 Abbildungen

Bibliographische Informationen der Deutschen Nationalbibliothek

Die Deutsche Nationalbibliothek verzeichnet diese Publikation in der Deutschen Nationalbibliographie; detaillierte bibliographische Daten sind im Internet über http://dnb.d-nb.de abrufbar.

ISBN 978-3-525-61425-9

(C) 2010 Vandenhoeck \& Ruprecht in Göttingen/www.v-r.de

Alle Rechte vorbehalten. Das Werk und seine Teile sind urheberrechtlich geschützt. Jede Verwendung in anderen als den gesetzlich zugelassenen Fällen bedarf der vorherigen schriftlichen Einwilligung des Verlags. Hinweis zu § 52a UrhG: Weder das

Werk noch seine Teile dürfen ohne vorherige schriftliche Einwilligung des

Verlages öffentlich zugänglich gemacht werden. Dies gilt auch bei einer entsprechenden Nutzung für Lehr- und Unterrichtszwecke.

Printed in Germany

Satz: Dörlemann Satz, Lemförde

Druck und Einband: CPI - Ebner \& Spiegel, Ulm

Gedruckt auf alterungsbeständigem Papier. 


\section{Inhalt}

Vorwort ..................... 7

\section{GRundFragen}

1. Religionswissenschaftliche Orientierung und theologische Positionierung (Bernd Schröder) . . . . . . . . . . . . .

2. Von der Fremdreligionen-Didaktik zum Interreligiösen Lernen (Rainer Lachmann) . . . . . . . . . . . . . . . . . . . . . . . . 26

3. Inhalt und Aufbau (Martin Rothgangel) . . . . . . . . . . . . . 41

\section{Ghristliche Konfessionen}

1. Evangelisch (Friedrich Schweitzer) . . . . . . . . . . . . 49

2. Freikirchlich (Walter Fleischmann-Bisten) . . . . . . . . . . . 63

3. Orthodox (Martin Tamcke) . . . . . . . . . . . . . . 83

4. Römisch-Katholisch (Albert Biesinger / Iris Gruhle) . . . . . . . . . 97

\section{Weltreligionen}

1. Judentum (Bernd Schröder) . . . . . . . . . . . . . . . . 113

2. Islam (Bernd Schröder) . . . . . . . . . . . . . . . . . . . 139

3. Hinduismus (fohannes Lähnemann) . . . . . . . . . . . . . . 164

4. Buddhismus (fohannes Lähnemann) . . . . . . . . . . . . . . 188 


\section{Sondergemeinschaften und neue Religionen / WELTANSGHAUUNGEN}

1. Zeugen Jehovas (Roland Biewald) . . . . . . . . . . . . . 217

2. Mormonen (Rainer Lachmann) . . . . . . . . . . . . . . 234

3. Anthroposophie / Christengemeinschaft (Christian Grethlein) . . . 254

4. Scientology (Andreas Grünschlo/s) . . . . . . . . . . . . . . . 274

\section{Moderne Variationen von Religion}

1. Patchwork-Religiosität / Synkretismen (Andrea Schulte) . . . . . 297

2. Kulturelle Transformationen von Religion (Manfred L. Pirner) . . 313

3. Zivilreligion (Rolf Schieder) . . . . . . . . . . . . 328

4. Fundamentalismus (Martin Rothgangel) . . . . . . . . . . . . 343

5. Esoterik / Okkultismus (Heinz Streib / Werner H. Ritter) . . . . . . 362

\section{Anhang}

1. Abkürzungen . . . . . . . . . . . . . . . . 383

2. Namenregister . . . . . . . . . . . . . . . 386

3. Sachregister . . . . . . . . . . . . . . . 393

4. Autorenverzeichnis . . . . . . . . . . . . . . 396 


\title{
2. Von der Fremdreligionen-Didaktik ZUM INTERRELIGIÖSEN LERNEN
}

\author{
RAINER LachmanN
}

Auf dem Hintergrund und im Horizont religionswissenschaftlicher Orientierung und theologischer Positionierung vollzog sich nach dem Zweiten Weltkrieg die konzeptionelle und didaktische Auseinandersetzung mit Christentum, Religionen und Religion, die hier in schwerpunktmäßiger Konzentration auf den schulischen RU in der Bundesrepublik Deutschland nachgezeichnet werden soll. In religionspädagogisch konzeptionellem Zusammenhang geht es dabei religionsdidaktisch um die Auswahl der Inhalte, ihre Begründung, Zielsetzungen und methodische Umsetzung in einer zusehends pluraler und globaler werdenden Welt und Gesellschaft, in der Lehrerinnen und Lehrer, Schüler und Schülerinnen lehren und lernen sollen. Gerade unter dem Blickwinkel schulischen RU werden Thematik und Problematik von Fremd- bzw. Weltreligionen zu einer beispielhaften Herausforderung im religionspädagogischen Umgang mit der vielperspektivischen Pluralität: der Pluralität der Religionen und Weltanschauungen, der politischen und wirtschaftlichen Verhältnisse, der Regionen und Bildungslandschaften sowie der wissenschaftlichen Bemühungen und Konzepte im Bereich schulischer Erziehung, Unterweisung und Bildung.

Der religionspädagogische Weg der letzten 50 Jahre mit seiner fragwürdigen Fokussierung auf einen konfessionellen bzw. christlichen RU in der öffentlichen Schule laut Art. 7,3 GG präsentiert dabei eine in theologischer, hermeneutischer und didaktischer Sicht äußerst aufschlussreiche Entwicklung, in der das religiös Fremde und Andere in zunehmendem Maße vom kaum beachteten Rand in den Mittelpunkt religionspädagogischen Interesses gerückt ist. Dieser religionsunterrichtliche Fortschritt verdankt sich sicher zu einem wesentlichen Teil der wachsenden Wahrnehmung und Ernstnahme der Voraussetzungen auf Schüler- wie Lehrerseite, in denen sich in mikrokosmischer Individualität und Sozialität die gesellschaftlichen Verhältnisse widerspiegeln. Sie lassen über den christlichen Glauben hinaus nach Religion und Religionen fragen und verlan- 
gen vom RU im Unterschied zu allen anderen Schulfächern undispensierbar die Auseinandersetzung mit der Wahrheitsfrage, die außer bei der Bescheidung auf objektiv-neutrale religionskundliche Deskription durchaus parteiische und konfessorische Beteiligung und Stellungnahme erlaubt. Das gerade macht das spannende Proprium religionsunterrichtlicher Beschäftigung mit fremden Religionen und anderer Religiosität aus und verordnet dem RU durchgängig die "Dauerreflexion", wie sich das Engagement für das Eigene mit der Toleranz und dem Respekt vor dem fremden Anderen verträgt und wie er sich demgegenüber in Theorie und Praxis verhält und religionspädagogisch konzeptionell und didaktisch ausweist. Stichworte wie Mission, Dialog, Begegnung signalisieren hier markante Problemstellen, die differenzierte und bedachte Betrachtung anmahnen. Dabei soll vom thematischen Zuschnitt des vorliegenden Bandes her nicht nur wie üblich den Weltreligionen, sondern wie ganz und gar unüblich auch den religiösen Sondergemeinschaften und Sekten religionsdidaktische Aufmerksamkeit zuteil werden. Der Boom an neuund pseudoreligiösen Bewegungen und Angeboten hat heute ein Ausmaß angenommen, das man sich vor 50 Jahren mit dem damaligen theologischen Abgesang auf Religion schlechterdings nicht vorstellen konnte was deshalb einmal mehr differenzierte historische Betrachtung erforderlich macht.

\subsection{Fremdreligionen im Unterricht vor der religionspädagogischen Wende}

Im konzeptionellen Horizont von »Evangelischer Unterweisung" (H. Kittel) und "Kirchlichem Unterricht" (K. Frör) waren Fremdreligionen kein didaktisch wirklich ernst genommenes Thema des RU. Sie blieben auch lehrplanmäßig ein marginaler 'Fremdkörper‘, dem - wenn überhaupt nur in der gymnasialen Oberstufe knapp bemessene Unterrichtszeit eingeräumt wurde. Paul Börgers Heft "Quellen zu den Religionen der Völker« (Heidelberg 1952) wurde in dieser Hinsicht für die fünfziger Jahre zum verbreitetsten Unterrichtsmaterial, das freilich seine starke Beeinflussung durch die Dialektische Theologie nicht verleugnen konnte und von daher eher unfreiwillig zum Zeugen dafür wurde, warum die Weltreligionen im konzeptionellen Kontext der Evangelischen Unterweisung religionsdidaktisch nicht Fuß fassen konnten. Rein theologisch war es das Verdikt, das Karl Barth über die Religion als Versuch des ungläubigen Menschen zur Selbsterlösung ausgesprochen hatte und das auch religionspädagogisch voll wirksam wurde. Sprechender Beleg für diese geradezu rallergische Aversion gegen "Religion« ist und bleibt Helmuth Kittels 
flammendes Plädoyer gegen die inhaltslose »Religion im Allgemeinen« mit seinem Schlachtruf »nie wieder Religionsunterricht!« « ${ }^{29}$ Dass ein solch fundamental negatives Urteil über Religion, mit dem die damalige Systematische Theologie nicht nur die Katechetik sfremd bestimmte, sondern auch die theologische Ausbildung beherrschte, das Thema der Weltreligionen aus der Theorie und Praxis der Evangelischen Unterweisung der fünfziger Jahre nahezu ausblendete, lag auf der Hand und bedingte dann auch die eher vereinzelt bleibenden Versuche eines Unterrichts über die Fremdreligionen an den Gymnasien. Hier verband sich die theologisch grundsätzliche Abwertung der Religionen mit überlegener christlicher Apologie, die dem Selbstanspruch der je anderen Religion kaum eine Chance zu gleichberechtigter und gleich geachteter Entfaltung bot.

Wenn Helmuth Kittel 1970 davon spricht, dass das Stoffgebiet der "fremden nichtchristlichen Religionen [...] seit langem einen festen Platz an unseren Höheren Schulen besitzt" und es ihm "heute in jeder Evangelischen Unterweisung unumgänglich" scheint, so gilt es hier offensichtlich einen religionsdidaktischen Meinungsumschwung zu konstatieren, der freilich konzeptionell erst richtig eingeordnet ist, wenn man Kittels gleichzeitige Anfragen an die Möglichkeiten wirklichen Verstehens einer Fremdreligion mithört und ernst nimmt. ${ }^{30}$

Im Überschneidungsfeld von kerygmatischem und hermeneutischem RU signalisiert 1961 ein Aufsatz Karl Ernst Nipkows über »Die Weltreligionen im Religionsunterricht der Oberstufe « 31 bemerkenswerte Zeitzeichen eines neu erwachenden und wachsenden Interesses an der ReligionenThematik. Von daher muss sich nach Nipkow der christliche Glaube nicht zuletzt vor der Jugend im Angesicht der Weltreligionen verantworten, und das kann weder durch eine rein vergleichende Gegenüberstellung geschehen, noch durch den scheinbar wissenschaftlichen Nachweis der Überlegenheit des Christentums. Gefragt sind stattdessen fundierte Kenntnis der Religionen und theologische Urteilsfähigkeit, was vom RU "sachliche Beschreibung und Untersuchung wie in allen anderen Unterrichtsfächern, zugleich aber existenzielles Einstehen" verlangt, »da es in diesem einen Fach um letzte Lebensfragen geht «. ${ }^{32}$ Hier partizipiert die Weltreligionen-Didaktik bereits an dem religionsunterrichtlich neuen hermeneutischen Paradigma existenzialer Interpretation und existenziellen Verstehens und erfuhr von daher zumindest ansatzweise eine Integra-

29 H. Kittel, Vom Religionsunterricht zur Evangelischen Unterweisung, Berlin / Hannover / Darmstadt (1947) ${ }^{31957,} 10$.

30 H. Kittel, Evangelische Religionspädagogik, Berlin 1970, 333.

31 In: EvErz 13/1961, 150-162.

32 K. E. Nipkow, aaO., 153. 
tion in den hermeneutischen RU und seinen auslegenden und verstehenden Umgang mit der christlichen Tradition.

Theologisch blieb Nipkow freilich in dieser Frühzeit mit seiner christozentrischen Betrachtungsweise noch mehr oder weniger dem Bannkreis der Dialektischen Theologie verhaftet. Zugleich verwies er mit seiner Forderung, dass »die unterrichtliche Besprechung der einzelnen Religionen" heute "nur noch mit dem Bezug auf die tatsächlichen Lebensfragen der Kirche in Mission und Ökumene" geschehen könne, auf den Ort, aus dem heraus die Beschäftigung mit den Fremdreligionen in der schulischen Unterweisung ihren Anfang nahm und ihre wesentlichen kirchlich theologischen Impulse empfing: auf die "missionarische Verantwortung der ganzen Kirche«, an der der Schüler mit seiner »Gliedschaft in der Gesamtkirche" teilhat. ${ }^{33}$

Nipkows Beitrag blieb nicht ohne Reaktion, sondern entfachte mit Heinz Röhrs Entgegnung bereits Anfang der sechziger Jahre eine erste religionsunterrichtlich motivierte Weltreligionen-Diskussion, die mit ihren Zweifeln an Nipkows Konzept bereits wichtige Problempunkte der künftigen "Auseinandersetzung mit den Fremdreligionen" ansprach. ${ }^{34}$ So stimmte Röhr zwar Nipkows Forderung zu, »daß bei der Bedeutung des Themas der Lehrer sich 'mit echtem pers. Engagement hat", fragte aber zugleich kritisch an, welchem »von uns Lehrern« unter den Voraussetzungen seines Studiums und später der Lehrpläne und Stundentafeln »ein tieferes Eindringen und Verstehen der fremdreligiösen Phänomene" so möglich sei, dass »die fremde Religion wirklich, deren 'Seele`, dem Schüler erschlossen werden « könne. ${ }^{35}$ Weiterführend relevant für die künftige Fremdreligionen-Didaktik war der von Röhr geforderte Aufbruch der Dialektischen Theologie hin zu anderen »Positionen innerhalb der Theologie der Ökumene und der Missionen«, die bei größtmöglicher Zurückhaltung gegenüber dem »bekennende(n) Zeugnis des Lehrers" sogar im Unterricht selbst erörtert werden sollten. ${ }^{36}$ Was sich hier angesichts verschiedener theologischer Positionen gleichsam als Dilemma zwischen abgelehnter "unbeteiligter 'Darbietung « und ebenso abgelehntem "zeugnishaftem Handeln « äußerte, ${ }^{37}$ offenbarte sich in der Folgezeit als undispensierbares Grundproblem jedweder religionsunterrichtlichen Beschäftigung mit anderen und fremden Religionen.

33 K. E. Nipkow, aaO., 155.

34 H. Röhr, Die Behandlung der Weltreligionen im Religionsunterricht der Oberstufe, in: Arbeitshilfe f. d. evgl. RU 13/1962, 19-24.

35 H. Röhr, aaO., $19 \mathrm{f}$.

36 H. Röhr, aaO., 21.

37 H. Röhr, aaO., 21. 
Das zeigte sich auch in den noch ganz im Kontext hermeneutischer Religionspädagogik beheimateten Ausführungen Gert Ottos zu den »Fremdreligionen im Unterricht", die in massiver Bestimmtheit mit der Behauptung eröffnet wurden, dass das "Verständnis unserer Welt [...] ohne Grundkenntnisse der großen Religionen kaum möglich « sei! ${ }^{38}$ Im Rahmen evangelischen RU müssten diese "grundsätzlich im Dialog mit biblischen Texten unterrichtet werden."Um dies zu erreichen, bedient sich der Unterricht »immer wieder des Vergleichs und der Gegenüberstellung mit biblischen Aussagen « - nicht wertend, sondern um »die jeweiligen Eigenarten am Gegenüber schärfer und präziser zu erfahren«.39 Freilich kann es der RU dabei nicht belassen, sondern muss sich im vergleichenden Vollzug biblischer Auslegung dem »Glaubensanspruch anderer Religionen" stellen. Er tut das in Verschränkung dialektischer und existenzialistischer Theologie über die didaktische und methodische Schiene der gemeinsamen »Grundsituation des Menschen« (diese geht »im IrdischDiesseitigen " nicht auf und gestaltet sich deshalb »selber Götter«), die bei "aller Verschiedenartigkeit" doch in allen fremden Religionen begegnet. Das biblische Zeugnis tritt dem in radikaler Andersartigkeit gegenüber und muss im Vergleich mit den Religionen »als allein angemessene Antwort " aufgewiesen werden, ${ }^{40}$ was in dieser dialektisch-theologischen $>\mathrm{Ab}$ solutheit< sicher leichter gesagt als religionsunterrichtlich getan war.

Bereits acht Jahre später sieht das denn auch beim selben Autor ganz anders aus; jetzt wird eine solche biblisch fixierte und dogmatisierte Befassung mit den Weltreligionen als "weithin apologetischer Natur« diffamiert und für eine primär religionskundliche und religionswissenschaftliche Auseinandersetzung mit den großen Weltreligionen - einschließlich Christentum - plädiert, deren leitendes Interesse religionskritischer Art ist. ${ }^{41}$

\subsection{Neuansätze in den 70er und 80er Fahren}

Die gesellschaftlichen Umbrüche und Aufbrüche im Kontext der sogenannten »68er « brachten auch für Religionspädagogik und RU eine radikale Wende, in der so gut wie alles, was vorher religionspädagogisch galt, von Grund auf in Frage gestellt wurde. Ob es nun die religionsunterricht-

38 G. Otto, Handbuch des Religionsunterrichts, Hamburg (1964) 21965, 116-121, bes. 117.

39 G. Otto, aaO., $118 \mathrm{f}$.

40 G. Otto, aaO., $119 \mathrm{f}$.

41 G. Otto / H. F. Dörger / F. Lott, Neues Handbuch des Religionsunterrichts, Hamburg 1972, 340-347, besonders 342. 
liche Mittelpunktstellung der Bibel, die Konfessionalität, das Verhältnis zu Staat und Kirche, die Inhalte oder die Methoden des aktuell praktizierten RU waren - alles wurde auf die gesellschafts- und religionskritische Waage gelegt, beurteilt, verurteilt und abgeurteilt. Dazu gehörte auch folgt man einem bekannten Flugblatt des Sozialistischen Lehrerbundes vom 4. November 1968 - die bisherige Beschäftigung mit den fremden Religionen im RU, an der kritisiert wurde, dass sie erst "frühestens in der Oberstufe des Gymnasiums, etwa 10. und 11. Klasse« erfolge und da auch nicht »umfassend und vorurteilsfrei« aufkläre, sondern - wie vom Lehrplan ausdrücklich vorgeschrieben - nur »einen allgemeinen Überblick [...] in aller Kürze vorsehe. "42 Welche religionsdidaktischen Folgen der Aufruf in diesem Punkt zeitigen konnte, zeigt etwa der oben angesprochene Versuch Gert Ottos und seiner Schüler, dem Thema Weltreligionen unter gesellschafts- und religionskritischem Aspekt religionsunterrichtlich gerecht zu werden.

Freilich sollte es noch knapp ein halbes Jahrzehnt dauern, bevor sich die rgewendeter Religionsdidaktik intensiver und bewusster mit den Weltreligionen befasste. Zunächst war sie vollends mit der Renaissance und Rezeption des allgemeinen Religionsbegriffs beschäftigt, den sie - pädagogisch an der Curriculumtheorie und theologisch u. a. an Paul Tillich orientiert - in die Religionspädagogik zurückholte. Damit wurde Religion zum einen individualisierend über die anthropologisch-ontologische, zum anderen gesellschaftsbezogen über die soziologische und ideologiekritische Dimension und Funktion wieder religionsunterrichtlich hoffähig. Hier liegen zweifelsohne wichtige Wurzeln für das, was im V. Teil dieses Bandes unter den »modernen Variationen von Religion« abgehandelt wird.

Anfang der 70er Jahre war es dann so weit: Die Weltreligionen gelangten zusehends ins Blick- und Interessenfeld einer Religionspädagogik, die im weiten Horizont didaktischer Problemorientierung ihr fruchtbares religionspädagogisches Wesen trieb. Es ist durchaus bemerkenswert, dass in diesem neuen didaktischen Kontext ein bayerischer Religionspädagoge unter den Ersten war, die sich mit mehreren Beiträgen zu "nicht christlichen Religionen im Unterricht« zu Wort meldeten (1971 ff.): Herkommend aus der Evangelischen Unterweisung und geprägt von seinen Unterrichtserfahrungen aus der gymnasialen Oberstufe äußerte sich $\mathrm{Hel}$ mut Angermeyer 1971 in der Zeitschrift "Mission« zu den Weltreligionen

42 Der Text des Flugblatts ist jüngst abgedruckt in R. Lachmann / B. Schröder (Hg.), Geschichte des evangelischen Religionsunterrichts in Deutschland. Quellen, Neukirchen-Vluyn 2009, $259 \mathrm{ff}$. 
und führte dabei weiter, was er bereits 1964 zur Frage "Mission und Weltreligionen in der Höheren Schule « begonnen hatte. ${ }^{43}$ Konnte er damals gleichsam als Kronzeuge für eine primär missionstheologisch orientierte und motivierte Beschäftigung mit den Religionen gelten, so wurde er jetzt Anfang der 70er Jahre zum Beispiel für ein Weiterdenken der Weltreligionen-Didaktik unter veränderten konzeptionellen Voraussetzungen und begleitete und bereicherte die erste Phase der neu entdeckten und bedachten Beschäftigung mit den Fremdreligionen im schulischen RU mit zahlreichen religionsdidaktischen Arbeiten. Er thematisierte schon 1974 die "Weltmacht Islam", noch früher das Verhältnis Christen und Juden und befasste sich 1975 in einem »Unterrichtsprojekt auch mit dem »Buddhismus im RU«. ${ }_{4}^{4}$ Damit setzte Angermeyer nicht nur wichtige Inhalte einer zukunftsfähigen Didaktik der Fremdreligionen bereits relativ früh unterrichtspraktisch um, sondern bemühte sich unter den Vorzeichen der Curriculumtheorie gleichzeitig darum, die Auswahl dieser Inhalte von den gesellschaftlichen Erfordernissen und politischen Realitäten, von den verschiedenen Voraussetzungen und Bedürfnissen auf Schülerseite sowie von den Anforderungen und Ansprüchen der zuständigen Fachwissenschaften Theologie und Religionswissenschaft her didaktisch umfassend zu begründen.

Dabei plädierte Angermeyer gegen die bisher üblichen allgemeinen Überblicke und mehr oder weniger schematischen Vergleiche für »eine ganzheitliche Sicht« der jeweiligen Religion, was eine solide kognitive Orientierung verlangte, ohne dabei der Illusion wertneutraler Information in einem vermeintlich objektiven Religions(kunde)unterricht aufzusitzen. In curricular leitender und integrierender Ausrichtung auf menschliche Lebenssituationen und -fragen konnte für ihn »die Auseinandersetzung mit Fremdreligionen nur im Dialog mit dem Christentum erfolgen«. Dies bedingt unseren »Kulturkreis« und bestimmte Angermeyers Position einer dezidiert evangelischen Religionspädagogik, die im Unterricht zu nicht-christlichen Religionen "auch immer eine Herausforderung" sah, "sich neu mit der eigenen Herkunft" aus dem christlichen Glauben zu beschäftigen. Dieser didaktisch wesentliche Akzent gehörte denn auch unaufgebbar zu Angermeyers "Zielbestimmung", wonach der RU »zum besseren Verständnis der Menschen anderen Glaubens verhelfen und zu einem entsprechenden Verhalten in der Begegnung mit ihnen

43 In: W. Ruf / E. Viering (Hg.), Die Mission in der Evangelischen Unterweisung, Stuttgart 1964, 375-384.

44 Vgl. die "Bibliographie Helmut Angermeyer« in R. Lachmann (Hg.), Religionsunterricht als religionspädagogische Herausforderung. FS Helmut Angermeyer z. 70. Geb. (Rh 9), Aachen 1982, 155-163. 
befähigen« sollte, was eben "nur in Korrelation mit den Inhalten und Lebensformen des christlichen Glaubens gewonnen werden " könnte. ${ }^{45}$

Wenn Herbert Schultze in einer Würdigung Angermeyers meinte, dessen Ziel der religionsunterrrichtlichen Beschäftigung mit Religionen »eigentlich nur Erläuterndes" hinzufügen zu müssen, ${ }^{46}$ so zeugt das aus der >vermittelnden Feder eines Mannes, der selbst zu den Pionieren einer neuen Weltreligionen-Didaktik nach der Wende zu zählen ist, zwar auch von dem innovatorischen Konsensgehalt, der sich in Angermeyers Vorstellungen äußert, zeigt aber vor allem die gemachten Fortschritte und bedachten Problemstände auf, die in den 70er Jahren für den RU süber Fremdreligionen bezeichnend waren. Schultzes "nur Erläuterndes" mit seinen "Beispielen aus Großbritannien, den Niederlanden und Schweden« öffnete dabei nicht nur den Blick über Deutschland hinaus, sondern ließ auch in didaktischer und konzeptioneller Hinsicht durchaus kontroverses Potenzial aufscheinen.

Schultze wusste darum, und als damaliger Direktor des Comenius-Instituts in Münster stellte er sich der Weltreligionen-Didaktik mit einschlägigen Tagungen und einer Vielzahl an Veröffentlichungen. Erste aus einer Tagung erwachsene Frucht war das von ihm zusammen mit Werner Trutwin herausgegebene Buch "Weltreligionen - Weltprobleme", das entschieden einen "neuen Ansatz von den Religionen selbst her « vertrat und in Verschränkung von "authentischen Äußerungen der Religionen" und »belangreichen Fragen" von Kindern und Jugendlichen einen "Dialog" zwischen "Menschen verschiedener Glaubensweisen" anstrebte, um darüber "zu einer Zusammenarbeit an Problemen unserer Zeit« zu gelangen. ${ }^{47}$ In problemorientierter Offenheit und curricularer »Lebensnützlichkeits-Perspektive" wurden so Folgerungen für den Unterricht über Religionen gezogen, an denen jetzt neben Theologie und Religionspädagogik auch die Religionswissenschaft eigenständig beteiligt war. Die »Religionen in ihrem Selbstverständnis $\mathrm{zu}$ verstehen und $\mathrm{zu}$ beurteilen« wurde fortan zu einem didaktisch unersetzlichen Programmpunkt religionsunterrichtlicher Weltreligionen - Didaktik und zum (an)gefragten Anspruch an die Religionswissenschaften. ${ }^{48}$

45 Helmut Angermeyer 7. 2. 1912. III. Fremdreligionen im Unterricht, in: B. Albers (Hg.), Religionspädagogik in Selbstdarstellungen I (Rh 6), Aachen 1980, 9-43/33-39, bes. 37.

46 H. Schultze, Unterricht über Religionen als Herausforderung des Religionsunterrichts, in: R. Lachmann (Hg.), aaO., 129-139, bes. 139.

47 H. Schultze, Beispiel eines Religionsgesprächs - Folgerungen für den Unterricht über Religionen, in: ders. / W. Trutwin ( $\mathrm{Hg}$.), Weltreligionen - Weltprobleme, Göttingen / Düsseldorf 1973, $23 \mathrm{ff}$.

48 H. Schultze, aaO., 24. 
Dem stellte sich ab Mitte der 70er Jahre nach dem Ende der »Sturmund Drangphase in der religionspädagogischen Diskussion um Religionswissenschaft - Religionskunde - >Weltreligionen im Unterricht « insbesondere $U d o$ Tworuschka, der unter leitendem religionswissenschaftlichem Interesse »den thematisch-problemorientierten Zugang zu den Religionen ermöglichen " wollte, um von daher »durch zuverlässige Informationen« über die Religionen im Unterricht traditionell verfestigte Einstellungen $\mathrm{zu}$ verunsichern" und "Denkanstöße zu vermitteln«.49 Das sollte vor allem durch eine faire, religionswissenschaftlich sachgemäße Interpretation von Texten ausgewählter Religionen im Unterricht erreicht werden, wobei das Verstehen der fremdreligiösen Texte immer zugleich »kommunikativ und kreativ sein« und die "Möglichkeit der Identifikation bieten" sollte..$^{50}$

Auf das hermeneutische und didaktische Problem, das sich mit der Behandlung von Fremdreligionen im konfessionellen RU stellte, ging Tworuschka an dieser Stelle nicht ein. Das mag an seinem kirchlich-konfessionell distanzierten problemorientierten Ansatz liegen, könnte aber auch mit seinem stark religionswissenschaftlich bestimmten Konzept zusammenhängen, dessen ’herkömmliche Nähe zur Religionskunde eine hermeneutische Reflexion über das Konfessorische im konfessionellen RU überflüssig machen würde. Dass mit der religionspädagogischen Wende auch dieses altbekannte religionskundliche Modell wieder seine Befürworter und Anhänger finden würde, war zu erwarten. »Vom Religionsunterricht zur Religionskunde« hieß denn auch die Forderung von Otto Thimme, der unter entschiedener Ablehnung des konfessionellen RU und seiner sinnlosen und sinnwidrigen dogmatischen Schranken einen "freien Unterricht in 'Religion und Weltanschauung « befürwortete. ${ }^{51}$ Ähnlich wie der Religionswissenschaftler Willi Cremer mit dem von ihm vertretenen "philosophisch-religionswissenschaftlichen Unterricht« 52 verlangte Thimme für die Etablierung dieses Faches einen "neu zu gründenden Fachbereich 'Religionswissenschaft « mit der Einrichtung diverser religionswissenschaftlicher Lehrstühle. ${ }^{53}$ An ihnen sollten die Voraussetzungen für einen religionskundlichen RU an den Schulen geschaffen werden, in dem möglichst objektiv und religiös neutral über die Religionen mit Einschluss des

49 U. Tworuschka, Religionen heute, München / Frankfurt/M. 1977, $7 \mathrm{ff}$.

50 U. Tworuschka, aaO., 11.

51 O. Thimme, Vom Religionsunterricht zur Religionskunde, in: $K$. Wegenast (Hg.), Religionsunterricht wohin?, Gütersloh 1971, 241-250, bes. 250.

$52 W$. Cremer, Schule und Religion. Überlegungen eines Religionswissenschaftlers, in: $\kappa$. Wegenast (Hg.), aaO., 225-240, bes. 239.

53 O. Thimme, aaO., 240. 
Christentums informiert werden sollte. Das wurde gleichsam zum religionskundlich funktionalen Grundbild, das bis heute mit immer wieder neuen Spielarten und Differenzierungen in der didaktischen Landschaft eines Unterrichts über die Welt- und Fremd-Religionen begegnet.

Es fand und findet sich nicht nur in religionskundlich gearteten RUModellen Großbritanniens oder Schwedens, sondern auch in Deutschland - und da vor allem in Gesetzen, Erlassen und Lehrplänen zum sogenannten Ethikunterricht, wo dieser zur Information über Religionen verpflichtet wird. Das zeigt sich am althergebrachten bayerischen Ethikunterricht ebenso wie am relativ neuen Brandenburger Unterricht "Lebensgestaltung - Ethik - Religionskunde«. Dieser trägt nicht nur in seinem Namen das Etikett "Religionskunde«, sondern verweist mit seinem Konzept zugleich auf verwandte Intentionen und Tendenzen in der jüngeren Religionspädagogik und ihrer Auseinandersetzung und Begegnung mit den nichtchristlichen Religionen in unserer Gesellschaft und Welt. ${ }^{54}$

Sicher nicht auf dem religionskundlichen Gleis, sondern eher in der von Angermeyer verfolgten Spur vollzog sich die Beschäftigung mit den Weltreligionen, die fohannes Lähnemann seit nunmehr 30 Jahren pflegt. Er war es, der 1977 die erste Monographie auf den Büchermarkt brachte, die sich mit "Nichtchristliche(n) Religionen im Unterricht. Schwerpunkt: Islam« befasste, und der dieser Untersuchung neun Jahre später zwei Bände »Weltreligionen im Unterricht" folgen ließ. In ihnen entwickelte Lähnemann an den "Fernöstlichen Religionen" und am "Islam" eine profilierte »theologische Didaktik für Schule, Hochschule und Gemeinde", die nicht nur als reife Frucht der nach der religionspädagogischen Wende einsetzenden Neubeschäftigung mit den Weltreligionen im RU gelten kann, sondern bereits auch Elemente in sich trägt, die für die neue interreligiös bestimmte Phase der Begegnung mit den Fremd-Religionen stehen.

Bilanzierend kann für die 70er und 80er Jahre des 20. Jh.s festgestellt werden, dass die didaktische Marginalität der Weltreligionen mit ihrer Beschränkung auf die oberen Klassen der Höheren Schulen überwunden ist und die Beschäftigung mit anderen Religionen jetzt für alle Schulstufen und Schularten für didaktisch notwendig und methodisch möglich erachtet wird. Lehrpläne, Schulbücher und diverses Unterrichtsmaterial zeugen davon. ${ }^{55}$ Als Inhalte solchen Unterrichts haben sich - relativ un-

54 Vgl. etwa Gert Ottos religionspädagogische Schriften ab den 90er Jahren: $R$. Lachmann, Wandlung und Aktualität: Gert Ottos Religionspädagogik 1980-1995, in: f. Henkys / B. Weyel (Hg.), Einheit und Kontext. FS P.C. Bloth (StTh 14), Würzburg 1996, 265-278.

55 Beispielhaft etwa das weit verbreitete "Religionsbuch für das 5./6. Schuljahr. Orientierung Religion« (Frankfurt/M. / Berlin / München 1973), dessen letztes Kapitel von den "anderen Religionen" handelt (130-140). 
abhängig von konzeptionellen Unterschieden - die großen Weltreligionen Islam, Hinduismus, Buddhismus und - im christlichen RU mit einer besonderen Profilierung - das Judentum etabliert; ${ }^{56}$ religiöse Sondergemeinschaften wie sogenannte Sekten sind so gut wie nirgends eigens verordnete Themenbereiche. Begründet wird die Behandlung der Weltreligionen im Unterricht argumentenreich und vielfältig konkretisiert und aktualisiert mit dem religiösen Pluralismus in unserer globalisierten Welt, was schulische Bildung undispensierbar zur Vermittlung von Grundkenntnissen der großen Religionen verpflichtet. Hier herrscht unter den Religionspädagogen quer durch alle Lager ebenso Einigkeit wie in der Ablehnung einseitig apologetisch-missionarischer Zielsetzungen. Einig ist man sich auch in dem Ziel, den Religionen in ihrem jeweiligen Selbstverständnis und ihren authentischen Glaubens- und Lebensformen in größtmöglicher religionswissenschaftlicher Sachgemäßheit gerecht zu werden. Unterschiedliche Akzentsetzungen im Blick auf Ziele des WeltreligionenUnterrichts sind primär konzeptionell-didaktisch bedingt und erwachsen aus der Gewichtung, die man der Konfessionalität des schulischen RU beimisst. Dessen ungeachtet partizipieren so gut wie alle Konzepte einer Weltreligionen-Didaktik am "Ziel, junge Menschen im Blick auf die nichtchristlichen Religionen dialogfähig zu machen" und sie im religionsunterrichtlichen »Themengebiet Weltreligionen ... für eine Situation der Begegnung auszurüsten«, was »nicht nur ein besseres Verständnis der religiösen und kulturellen Wurzeln des anderen, sondern auch des eigenen Glaubens" bewirken könne. ${ }^{57}$ Auch in Bezug auf diese so allgemein formulierte religionsdidaktische Hoffnungsperspektive, die uns ansatzweise schon bei Angermeyer begegnete, dürfte unter den Religionspädagogen relativer Konsens herrschen, zumal dieses wesenhaft dialogbezogene Lernen der Weltreligionen über die inhaltliche Dimension hinaus auch großes methodisches Potenzial besitzt und freisetzen kann.

56 Vgl. etwa die einschlägigen Artikel in gängigen religionspädagogischen Kompendien und Handbüchern wie z.B.: G. Adam / R. Lachmann (Hg.), Religionspädagogisches Kompendium, Göttingen (1984) 62003, 427-467; G. Bitter / R. Englert / G. Miller / K.E. Nipkow (Hg.), Neues Handbuch religionspädagogischer Grundbegriffe, München 2002, 283-291; P. Schreiner / U. Sieg / V. Elsenbast (Hg.), Handbuch Interreligiöses Lernen, Gütersloh 2005, 134-167; R. Lachmann / R. Mokrosch / E. Sturm (Hg.), Religionsunterricht - Orientierung für das Lehramt, Göttingen 2006, 317-352.

57 f. Lähnemann, Zugänge zu den Weltreligionen, in: G. Adam / R. Lachmann (Hg.), aaO., 323-339 (1984), bes. 336 und f. Lähnemann, Weltreligionen im Unterricht. Teil II: Islam, Göttingen 1986, 14. 


\subsection{Von der Weltreligionen-Didaktik zum Interreligiösen Lernen}

Mit gleitenden Vor- und Übergängen gerät etwa seit Ende der 80er Jahre des vorigen Jh.s "Interreligiöses Lernen" (IL) zusehends in den Blickpunkt der Religionspädagogik und etabliert sich dort als wirkmächtiger Terminus technicus, der aus der aktuellen religionspädagogischen Diskussion nicht mehr wegzudenken ist und einen gleichsam multiplen Perspektivwechsel im Umgang mit den Religionen signalisiert. Dieser ist sicher insofern von der herkömmlichen Behandlung von Weltreligionen im RU - ihren svermeintlichen Siegeszug konnten wir für die 70er und 80er Jahre verfolgen - zu unterscheiden, als IL im Sinne eines Lernprinzips $^{58}$ bzw. einer "Lerndimension «59 die Konzentration auf den RU überschreitet in Richtung auf die Schule als Ganze und andere Lernorte, an denen Begegnung und Dialog mit fremden und anderen Religionen zu lernen und zu leisten sind.

Dabei kann allerdings keinesfalls von einer "strikten" Unterscheidung zwischen der »traditionellen Behandlung von Weltreligionen im RU« und IL die Rede sein. ${ }^{60}$ Denn Letzteres verdankt sich nicht nur in vieler Hinsicht der religionsunterrichtlichen Weltreligionen-Didaktik, sondern bleibt auch bis heute - über alle konzeptionellen Unterschiede hinweg essenzieller Bezugspunkt allen religionspädagogisch einschlägigen Nachdenkens. Das gilt für fohannes Lähnemanns dezidiert "Evangelische Religionspädagogik in interreligiöser Perspektive« ebenso wie für die Vorstellungen der katholischen Religionspädagogen H.-G. Ziebertz und St. Leimgruber zum "religionsdidaktischen Prinzip« des IR oder das Hamburger interreligiöse Konzept eines "Religionsunterrichts für alle«. Selbst $F$. Rickers, der in seinem Artikel der strikten Unterscheidung von Weltreligionen-RU und IL das Wort redet, handelt im Wesentlichen von IL in Schule und RU.

Freilich verbirgt sich hinter Rickers rigoroser Trennungsposition eine grundsätzliche Problematik des IL, auf die Karl Ernst Nipkow mit Recht hinweist. ${ }^{61}$ Zwar gesteht Rickers dem Nürnberger Konzept Lähnemanns $\mathrm{zu}$, dass es einen »optimal verbesserten traditionell stofforientierten Un-

58 G. Hilger / St. Leimgruber / H.-G. Ziebertz ordnen in ihrer "Religionsdidaktik « (München 2001, 303 u. 433-442) "Interreligiöses Lernen" unter "Religionsdidaktische Prinzipien" ein.

59 Für F. Rickers ist "Interreligiöses Lernen« eine "Lerndimension" (vgl. seinen Art. "Interreligiöses Lernen« in: LexRP I, Neukirchen-Vluyn 2001, Sp. 874-881, bes. 875).

60 F. Rickers, aaO., 875.

61 K. E. Nipkow, Ziele interreligiösen Lernens als mehrdimensionales Problem, in: V. Elsenbast / P. Schreiner / U. Sieg (Hg.), aaO., 362-380, bes. $364 \mathrm{ff.}$ 
terricht über Weltreligionen" vertrete, kommt aber darüber hinaus zu dem Schluss, dass "hier kaum von >dialog. $u$. sinterrel.` gesprochen werden kann", weil aus dem IL bei Lähnemann »die Warte christl. Theologie u. RP « spreche. ${ }^{62}$ Nun ist das sicher insofern richtig, als damit auf das (religions-)pädagogische Handicap des konfessionellen RU an der Schule verwiesen wird, das einen RU im Klassenverband verhindert und von daher echter interreligiöser Begegnung im RU gleichsam leibhaftig im Wege steht. Demgegenüber hat das Hamburger Modell eines »RU für alle", das von F. Doedens und W. Weifse vertreten ${ }^{33}$ und von Rickers favorisiert wird, eindeutig ein Prae. Es fordert als schulischen »Beitrag zur religiösen Alphabetisierung« und »Forum für die Weltreligionen« einen »für alle Schülerinnen und Schüler gemeinsamen dialogischen Religionsunterricht", der "von einer direkten Beeinflussung für eine bestimmte Konfession, Religion oder Weltanschauung abzusehen" hat. ${ }^{64}$

Bei aller auch theologisch-ökumenisch begründeten Berechtigung der Dialogdimension des Hamburger Konzepts bleibt die für diesen RU ebenfalls beanspruchte Dimension der »Wahrheit« fragwürdig, vor allem wenn er sich ausdrücklich von einem »religionskundlich-neutralen« Unterricht absetzt und ohne »direkte Beeinflussung « »ür einen dialogischen Religionsunterricht für alle in evangelischer Verantwortung und in ökumenischer Offenheit« eintritt. ${ }^{65}$ Gerade in dieser Beziehung scheint das Hamburger Modell "noch einigermaßen vage und unbestimmt zu sein«. ${ }^{6} 6$ Kann man doch einem RU wohl kaum das Prädikat IL absprechen, nur weil seine Beschäftigung mit den Fremdreligionen nicht im Klassenverband und dezidiert aus christlicher Warte geschieht. Das hieße die "wichtigste Frage nach der elementaren Wahrheit « in und aus konfessorischer Parteilichkeit religionsdidaktisch zu verdrängen und darüber der »wertenden Auswahl « und Vermittlung im interreligiösen Elementarisierungsund Lernprozess nicht genügend Aufmerksamkeit und Gewicht beizumessen. ${ }^{67}$

62 F. Rickers, aaO., 880.

63 Vgl. n.a. F. Doedens / W. Weifse (Hg.), Religionsunterricht für alle. Hamburger Perspektiven zur Religionsdidaktik, Münster u.a. 1997.

$64 W$. Weise, Leitsätze für einen dialogischen und ökumenischen Religionsunterricht für Alle in Hamburg, in: ders. (Hg.), Wahrheit und Dialog, Münster u.a. 2002, 121-143, bes. $124 \mathrm{f}$.

65 W. Weise, aaO., 130.

66 H. Rupp, "Religionsunterricht für alle in evangelischer Verantwortung und in ökumenischer Offenheit - was soll und kann dies heißen?, in: W. Weisse (Hg.), aaO., 145-151, bes. 149.

67 R. Boschki / C. Schlenker, Brücken zwischen Pädagogik und Theologie. Mit Karl Ernst Nipkow im Gespräch, Güterloh 2001, 114; vgl. R. Lachmann, Problemorientierter Religionsunterricht, Elementarisierung und die Frage nach einem Kerncurriculum, in: $F$. 
Hier fragt es sich etwa, ob man sich mit der »individuelle(n) Anerkennung « jedweder geäußerten religiösen Auffassung und damit verbunden der "Anerkennung von Differenz" auf Seiten der Schüler und Schülerinnen, wie von Barbara Asbrand mit ihrem dem Hamburger Modell folgenden Konzept vertreten, ${ }^{68}$ als allein leitenden didaktischen Kriterien für IL im (christlichen) RU wirklich bescheiden kann, ja darf. Wird durch einen solchen pädagogisch motivierten absolut gesetzten Maßstab die Inhaltsdimension nicht derart relativiert, dass selbst die abstrusesten und menschenverachtendsten religiösen Vorstellungen, wie sie etwa von Sektenangehörigen in den RU mit- und eingebracht werden können, tolerant akzeptiert werden müssen? Letzteres ist nicht nur eine Frage, die sich in der bisher sträflich vernachlässigten interreligiösen Auseinandersetzung mit der modernen 'Sektenszener stellt, sondern im aufgezeigten Fragenhorizont auch ein hermeneutisches und didaktisches Grundproblem des IL überhaupt. Bei allem religionspädagogischen Fortschritt, den man dem IL in seiner Öffnung zum interkulturellen Lernen und seiner Differenzierung nach Lernorten, Lernanlässen und Zielen und den am Lernprozess Beteiligten attestieren muss, bleibt das auch fürderhin elementare Aufgabe für alles Lernen an, mit und durch Fremdreligionen.

Gegenüber dem Abseitigen, Fernen und Marginalen schließlich, das in der Religionspädagogik der 50er und 60er Jahre des vorigen Jh.s dem Begriff "Fremdreligionen" anhaftete, erfuhr dieser Terminus im Kontext IL eine ganz neue, eigene und wesentliche Dignität, die nicht zuletzt auch damit zusammenhängt, dass das Christentum selbst in weiten Teilen unserer säkular pluralistischen Gesellschaft zu etwas Fremden geworden ist. Dadurch gewinnt die mit dem IL religionsunterrichtlich vollzogene »hermeneutische Kehre« 69 vom Verstehen des Eigenen zum Verstehen des Fremden noch eine zusätzliche gewissermaßen reziproke Akzentuierung, und wird das »Lernen an Differenzen ${ }^{70}$ an Fremdem und Andersartigem zur komplexen didaktischen und methodischen Herausforderung, mit der für IL im RU ein äußerst fruchtbares Spannungsfeld eröffnet ist, das der jüngsten Fremdreligionen-Didaktik wichtige Impulse vermittelt hat und vermitteln kann.

Schweitzer / V. Elsenbast / Chr. T. Scheilke (Hg.), Religionspädagogik und Zeitgeschichte, Gütersloh 2008, 28-40, bes. $37 \mathrm{f}$.

68 B. Asbrand, Zusammen Leben und Lernen im Religionsunterricht, Frankfurt/M. 2000, $244 \mathrm{f}$.

69 K. E. Nipkow, Schule und Gesellschaft im religiösen Pluralismus - Voraussetzungsprobleme und Ziele interreligiösen Lernens, in: GVEE Informationen Mai 1993 / 2, 5-34, bes. 14 .

70 G. Hilger / St. Leimgruber / H.-G. Ziebertz, aaO., 438. 
Anregend ist in dieser Beziehung z.B. Heinz Streibs Ansatz einer "xenosophischen Religionsdidaktik", die mittels der "Kultivierung von Fremdheit" die Fremdheitserfahrungen im Umgang mit der unterrichteten Religion produktiv nutzen will "als Herausforderung, als widerständiger Eigen-Sinn und als Anlass für Neugierde und Lernbegier«. Das schließt ein, »dass die fremde Religion auch fremd sein darf «71 und man ihre »bleibende Fremdheit zu respektieren" hat. ${ }^{72}$ Zugleich will Streib mit solcher 'Be-Fremdungsarbeit< verhindern, dass das religionsunterrichtliche Fragen nach dem Gemeinsamen von eigener und fremder Religion die Fremdheitserfahrungen zu stark reduziert, anstatt sie zu »kultivieren«. Hier gerät die xenosophische Religionsdidaktik an ihre Grenzen und in die Gefahr, der Fremdheit im interreligiösen Lernprozess zu viel an didaktischer Eigengewichtigkeit zuzumessen und darüber das "Gemeinsame inmitten des Differenten « didaktisch zu gering zu achten. ${ }^{73}$

Rückblickend wie vorausschauend kann so die Doppelsicht des Perspektivwechsels dem IL dazu verhelfen, nicht nur die fremde Religion mit den Augen des anderen wie den eigenen zu sehen, sondern auch die eigene Religion aus eigener und aus fremder Sicht rwahrzunehmen dürfte im heutigen christlichen RU das Fremde und Befremdliche an der vorgeblich eigenen und vertrauten Religion der Kinder und Jugendlichen eher zunehmen. Nicht mehr im Brecht'schen Sinne Verfremdung einer altbekannten und gewohnt christlichen Religion ist jetzt zuerst gefragt, sondern Befremdungsarbeit an der fremd gewordenen, angeblich vertrauten eigenen Religion. Da könnte sich für die Zukunft des IL im schulischen RU ein neuer religionsdidaktischer Horizont auftun, der insbesondere bei der Beschäftigung mit Konfessionen, Weltreligionen, Sondergemeinschaften und modernen Variationen von Religion verstärkte Beachtung verdient und verlangt und nicht zuletzt bei der Auseinandersetzung um die Wahrheitsfrage stets mit bedacht sein will.

$71 H$. Streib, Wie finden interreligiöse Lernprozesse bei Kindern und Jugendlichen statt? Skizze einer xenosophischen Religionsdidaktik, in: V. Elsenbast / P. Schreiner / U. Sieg (Hg.), aaO., 230-243, bes. 231 u. 241.

72 G. Hilger / St. Leimgruber / H.-G. Ziebertz, aaO., 440.

73 K. E. Nipkow, Ziele interreligiösen Lernens als mehrdimensionales Problem, in: V. Elsenbast / P. Scheiner / U. Sieg (Hg.), aaO., 362-380. 